\title{
Zernike phase spatial filter for measuring the aberrations of the optical structures of the eye
}

\author{
Svetlana N. Khonina ${ }^{1 *}$, Victor V. Kotlyar ${ }^{2}$, Dmitriy V. Kirsh ${ }^{2}$ \\ ${ }^{1}$ Image Processing Systems Institute of the Russian Academy of Sciences, 151 Molodogvardejskaya st., Samara \\ 443001, Russian Federation \\ ${ }^{2}$ Samara State Aerospace University, 34 Moskovskoe shosse, Samara 443086, Russian Federation \\ *e-mail: khonina@smr.ru
}

\begin{abstract}
To measure directly the wavefront aberration coefficients, we propose to use the multi-order diffractive element fitted with the set of Zernike polynomials. Polynomials of lowest degree describe defocusing (ametropy) and astigmatism. Coefficients of highest degree correspond to the spherical aberration of oblique rays that occurs as a consequence of misalignment of the crystalline lens and foveola, as well as deflection at the periphery of the crystalline lens. Multi-order elements allow several tens of expansion coefficients to be measured simultaneously, which will enable to investigate insufficiently known high-order aberrations for the differentiated diagnostics of eye diseases. (C) 2015 Samara State Aerospace University (SSAU).
\end{abstract}

Keywords: wavefront aberrations, eye optical system, Zernike polynomials, multi-order diffractive elements, expansion coefficients.

Paper \#2468 received 2015.05.29; revised manuscript received 2015.06.23; accepted for publication 2015.06.25; published online 2015.06.30.

\section{References}

1. M. S. Smirnov, "Measurement of the wave aberration of the human eye," Biofizika 6, 776-795 (1961).

2. H. C. Howland, and B. Howland, "A subjective method for the measurement of monochromatic aberrations of the eye,” J. Opt. Soc. Am. 67(11), 1508-1518 (1977).

3. F. Berny, and S. Slansky, "Wavefront determination resulting from Foucault test as applied to the human eye and visual instruments," Optical Instruments and Techniques, 375-386 (1969).

4. P. Artal, J. Santamaría, and J. Bescós, "Retrieval of the wave aberration of human eyes from actual pointspread function data,” J. Opt. Soc. Am. 5(8), 1201-1206 (1988).

5. D. A. Atchison, "Invited review recent advances in measurement of monochromatic aberrations of human eyes," Clin Exp Optom 88(1), 5-27 (2005).

6. A. S. Goncharov et al., "Modal tomography of aberrations of the human eye," Laser Physics, 16(12), 16891695 (2006).

7. M. Lombardo, and G. Lombardo, "New methods and techniques for sensing the wave aberrations in human eyes," Clin Exp Optom 92(3), 176-186 (2009).

8. P. Artal, "Optics of the eye and its impact in vision: a tutorial," Advances in Optics and Photonics 6(3), 340367 (2014).

9. G. Artzner, "Microlens arrays for Shack-Hartmann wavefront sensors," Opt. Eng. 31(6), 1311-1322 (1992).

10. J. Liang et al., "Objective measurement of the WA's aberration of the human eye with the use of a HartmannShack sensor," J. Opt. Soc. Am. 11, 1949-1957 (1994).

11. American National Standards Institute, Inc. American National Standards for Ophthalmics - Methods for Reporting Optical Aberrations of Eyes. ANSI Z80.28 (2004).

12. International Organization for Standardization (ISO). Ophthalmic Optics and Instruments - Reporting Aberrations of the Human Eye. Geneva, Switzerland (2008).

13. R. A. Applegate et al., "Visual acuity as a function of Zernike mode and level of root mean square error," Optom Vis Sci 80(2), 97-105 (2003). 
14. D. L. Golovashkin et al., Computer Design of Diffractive Optics, V. A. Soifer (Ed.), Cambridge Inter. Scien. Pub. Ltd.\& Woodhead Pub. Ltd., Cambridge (2012).

15. S. N. Khonina et al., "Experimental selection of spatial Gauss-Laguerre modes," Optical Memory and Neural Networks 9(1), 69-74 (2000).

16. V. V. Koltyar, and S. N. Khonina, "Multi-order diffractive optical elements to process data," Chapter 2 in Perspectives in Engineering Optics, K. Singh, V. K. Rastogi (Eds.), Anita Publications, Delhi, 47-56 (2003).

17. S. N. Khonina et al., "Generation and selection of laser beams represented by a superposition of two angular harmonics," Journal of Modern optics, 51(5), 761-773 (2004).

18. V. V. Kotlyar et al., "Coherent field phase retrieval using a phase Zernike filter," Computer Optics 17, 43-48 (1997).

19. S. N. Khonina et al., "Phase reconstruction using a Zernike decomposition filter," Computer Optics 18, 52-56 (1998).

20. S. N. Khonina, V. V. Kotlyar, and Ya Wang, "Diffractive optical element matched with Zernike basis," Pattern Recognition and Image Analysis 11(2), 442-445 (2001).

21. M. Born, and E. Wolf, Principlies of Optics, Pergamon Press, Oxford (1968).

\section{Introduction}

Wave aberrations of the eye can be measured using various subjective and objective methods. The subjective methods are based on the collection of the patient's visual responses, while the objective methods do not require the cooperation with the patient and are based on the image recording after the double-pass through the ocular lens. Among the methods developed over the years, the following methods can be singled out: the Vernier method - alignment [1], aberroscopy [2], the Foucault knife-edge method [3] and the method for phase retrieval from the retina image [4].

Existing clinical aberrometers provide sufficiently accurate measurements of deflection of the eye's wavefront [5-8]. In this case high-order aberrations are measured to assess the personal deflections of the wavefront, including those related to the professional activity or age-related changes, in order to optimise the optical (with contact or intraocular lenses) or surgical correction of the human eye.

Currently, the most widely used method for the measurement of the eye aberration is the HartmannShack wavefront sensor $[9,10]$. The sensor consists of a microlens array optically conjugated to the pupil and a camera located in the focal plane of the microlens array. If a plane wavefront reaches the microlenses then the perfectly regular grid of light spots is formed on the camera. However, if the wavefront is distorted by aberrations, the light spots are located irregularly. The displacement of each spot from the reference position is proportional to the wavefront derivative in each microlens area. Thus, the wavefront is reconstructed from the displacements of the light spots recorded by the Hartmann-Shack sensor, and then the aberrations are calculated.

A common representation of the wavefront is a set of Zernike polynomials, which correspond to different wavefront aberrations [11, 12]. Coefficients for the expansion of the wavefront in orthogonal Zernike polynomials make it possible to determine the root mean square error of the deflection from an ideal wavefront. The coefficients with high absolute value automatically indicate to the aberrations that distort the wavefront in the highest degree and consequently allow to significantly speed up and simplify the analysis of patient's vision [13].

In this paper, we propose to use diffractive optics methods for the direct optical measurement of amplitudes of coefficients for the expansion of the individual visual system wavefront in Zernike polynomials [14].

The diffractive optical elements (DOE) forming simultaneously several laser beam modes in different diffraction orders (so-called multi-order or multichannel elements) are successfully used as a spatial filters for the analysis of a set of laser beam transverse modes [14-17].

Similar multi-order DOE fitted with the set of Zernike polynomials can be used in the wavefront analysis and reconstruction [18-20].

In this paper, wavefront aberrations are analysed using a phase spatial filter, with orthogonal circular Zernike polynomials used as a basis of the light field expansion. Note that in this case, not the phase field but the complex amplitude is expanded into the Zernike basis, with the intensity generated in the spatial plane of Fourier-spectrum and proportional to the field expansion coefficients. The coefficient modules measured are then used for computing the argument of the light field complex amplitude. We are also concerned with iterative algorithms for computing the Zernike filter phase and the phase of light field complex amplitude.

\section{Theoretical bases}

There is a complete set of orthogonal functions with angular harmonics in a circle of radius $r_{0}$. These are the circular Zernike polynomials [21]:

$$
\Psi_{n m}(r, \varphi)=A_{n} R_{n}^{m}(r) \exp (\operatorname{im} \varphi)
$$

where 


$$
A_{n}=\sqrt{\frac{n+1}{\pi r_{0}^{2}}},
$$

$$
\begin{aligned}
&(-1)^{p}(n-p) ! \times \\
& R_{n}^{m}(r)=\sum_{p=0}^{(n-m) / 2} \times\left[p !\left(\frac{n+m}{2}-p\right) !\left(\frac{n-m}{2}-p\right) !\right]^{-1} \times \\
& \times\left(\frac{r}{r_{0}}\right)^{n-2 p},
\end{aligned}
$$

$R_{n}^{m}(r)$ are the radial Zernike polynomials:

$$
\begin{aligned}
& R_{n}^{-m}(r)=R_{n}^{m}(r), \quad R_{n}^{ \pm 1}\left(r_{0}\right)=1, \\
& R_{2 k+1}^{2 l}(r)=0, \quad R_{2 k}^{2 l+1}(r)=0, \quad|m| \leq n, \quad R_{0}^{0}(r)=1,
\end{aligned}
$$

and $(r, \varphi)$ are polar coordinates.

The expansion of the light field with complex amplitude $E(r, \varphi)$ into a series in terms of the functions in Eq. (1) is given by

$$
\begin{aligned}
& E(r, \varphi)=\sum_{n=0}^{\infty} \sum_{m=-n}^{n} C_{n m} \Psi_{n m}(r, \varphi), \\
& \int_{0}^{r_{0}} R_{n}^{m}(r) R_{p}^{m}(r) r \mathrm{~d} r=\frac{r_{0}^{2}}{2(n+1)} \delta_{n p}, \\
& C_{n m}=\int_{0}^{r_{0}} \int_{0}^{2 \pi} E(r, \varphi) \Psi_{n m}^{*}(r, \varphi) r \mathrm{~d} r \mathrm{~d} \varphi .
\end{aligned}
$$

In the plane of a spatial Fourier-spectrum that may be generated by a spherical lens of focal length $f$, the light field complex amplitude $F(\rho, \theta)$ takes the form

$$
\begin{aligned}
& F(\rho, \theta)=\frac{k}{2 \pi f} \times \\
& \times \int_{0}^{r_{0} 2 \pi} E(r, \phi) \exp \left[-i \frac{k}{f} r \rho \cos (\phi-\theta)\right] r \mathrm{~d} r \mathrm{~d} \phi,
\end{aligned}
$$

where $k=2 \pi / \lambda$ is the wavenumber of light, $\lambda$ is the wavelength, and $(\rho, \theta)$ are the polar coordinates. Based on Eq. (2), the light field expansion, Eq. (4), in terms of the Zernike polynomials of Eq. (1) is given by

$$
\begin{aligned}
& F(\rho, \theta)= \\
& =\frac{k}{f} \sum_{n=0}^{\infty} \sum_{m=-n}^{n}(-i)^{m} C_{n m} A_{n m} e^{i m \theta} \int_{o}^{2 \pi} R_{n}^{m}(r) J_{m}\left(\frac{k}{f} r \rho\right) r \mathrm{~d} r .
\end{aligned}
$$

In deriving Eq. (5), we made use of the integral representation of the Bessel functions of the first kind and $m$-th order:

$$
J_{m}(x)=\frac{i^{m}}{2 \pi} \int_{0}^{2 \pi} \exp (-i x \cos t+i m t) \mathrm{d} t
$$

The integral in Eq. (5) may be taken explicitly [21]:

$$
\begin{aligned}
& W_{n m}(\rho)=\int_{0}^{r_{0}} R_{n}^{m}(r) J_{m}\left(k f^{-1} r \rho\right) r \mathrm{~d} r= \\
& =(-1)^{(n-m) / 2} r_{0}^{2} \frac{J_{n+1}\left(k f^{-1} r_{0} \rho\right)}{\left(k f^{-1} r_{0} \rho\right)} .
\end{aligned}
$$

From Eq. (6), one can see that at $n>0$ the complex amplitude at central points $\rho=0$ is equal to zero:

$$
W_{n m}(\rho=0)=\left\{\begin{array}{c}
0, n>0, \\
\frac{r_{0}^{2}}{2}, n=0 .
\end{array}\right.
$$

Hence, at $n>0$ the intensity distribution in diffraction orders of the Fourier-plane will be circular in structure.

An optical configuration of the spectral Zernike analyser to illustrate the use of a phase Zernike filter in analysis of the wavefront of amplitude $E(r, \varphi)$ is shown in Fig. 1. Similar to the Hartman-Shack wavefront sensor $[9,10]$, the Zernike filter is mounted directly in the plane of the wavefront to be studied, with a spherical lens $L$ of focal length $f$ placed immediately behind it. A photoreceiver array matched to the computer PC is placed in the rear focal plane of the lens L.

In our formulation of the problem, lens aberrations are not included. We believe they will be substantially less than the analysed aberrations of an eye.

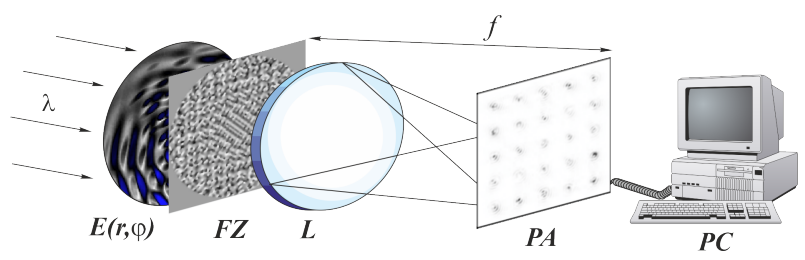

Fig. 1 Optical configuration of the Zernike analyser: ZF is the Zernike filter, $\mathrm{L}$ is a spherical lens, PA is a photoreceiver array, and $\mathrm{PC}$ is a computer.

\section{Design of the phase-only multi-order Zernike filter}

In order for the transmission function of the $\mathrm{ZF}$ to be phase-only:

$$
\tau(r, \varphi)=\exp [i S(r, \varphi)]
$$


it should be sought for in the form

$$
\begin{aligned}
& \tau(r, \varphi)= \\
& =\sum_{n=0}^{N} \sum_{m=-n}^{n} \times \exp \left[i k f^{-1} r \rho_{n m} \cos \left(\varphi-\theta_{n m}\right)+v_{n m}\right],
\end{aligned}
$$

where $\left(\rho_{n m}, \varphi_{n m}\right)$ are the vectors of the carrier spatial frequencies in polar coordinates and $v_{n m}$ are the free parameters of the task to be fitted in such a manner as to make Eq. (8) an exact equality. Once the light intensity proportional to the squared modulus of the expansion coefficients in Eq. (2),

$$
I_{n m}=\left|C_{n m}\right|^{2}
$$

has been measured at discrete points of the Fourierplane (see Fig. 1), one must perform additional computation in order to find the light field phase from Eq. (2):

$$
Q(r, \varphi)=\arg E(r, \varphi) .
$$

To do this, one may use an algorithm similar to the algorithm of Eqs. (3) and (4) and find the phase estimate of light field in the $(k+1)$-th iteration in the form

$$
\begin{aligned}
& Q_{k+1}(r, \varphi)= \\
& =\arg \left\{\sum_{n=0}^{N} \sum_{m=-n}^{n} \sqrt{I_{n m}} \Psi_{n m}(r, \varphi) \exp \left[i \vee_{n m}^{(k)}\right]\right\},
\end{aligned}
$$

where $v_{n m}^{(k)}$ are the free parameters in the $k$-th iteration derived from the equation

$$
v_{n m}^{(k)}=\arg \left\{\int_{0}^{r_{0}} \int_{0}^{2 \pi} \exp \left[i Q_{k}(r, \varphi)\right] \Psi_{n m}^{*}(r, \varphi) r \mathrm{~d} r \mathrm{~d} \varphi\right\}
$$

where $Q_{k}(r, \varphi)$ is the sought-for phase estimate in the $k$-th iteration.

Since the wavefront aberrations met with in optical systems are described by even functions relative to the azimuth angle $\varphi[21]$, the wave field $E(r, \varphi)$ may be written as

$$
E(r, \varphi)=\exp \left\{i \sum_{n=0}^{N} \sum_{m=-n}^{n} B_{n m} R_{n}^{m}(r) \cos (m \varphi)\right\} .
$$

In this connection, instead of the general expansion in Eq. (9) one should use the expansion in terms of the even functions

$$
\begin{aligned}
& E(r, \varphi)=\sum_{n=0}^{\infty} \sum_{m=-n}^{n} C_{n m} \bar{\Psi}_{n m}(r, \varphi), \\
& \bar{\Psi}_{n m}(r, \varphi)=\varepsilon_{m} \sqrt{\frac{n+1}{\pi r_{0}^{2}}} R_{n}^{m}(r) \cos (m \varphi),
\end{aligned}
$$

where $\varepsilon_{m}=\left\{\begin{array}{c}2, m \neq 0 \\ 1, m=0\end{array}\right.$

For small aberrations, the relation between the expansion coefficients $B_{n m}$ and $C_{n m}$ is linear

$$
1+i B_{00}=\frac{C_{00}}{\sqrt{\pi r_{0}^{2}}}, i B_{n m}=\varepsilon_{m} \frac{C_{n m}}{\sqrt{\pi r_{0}^{2}}} .
$$

For arbitrary aberrations, the relation between $B_{n m}$ and $C_{n m}$ is non-linear and on measuring the modules $\left|C_{00}\right|^{2}$ one has to use the algorithm of Eqs. (11) and (12) in order to derive the phase $Q(r, \varphi)$ of Eq. (10). Then, using Eq. (13) one derives the wave aberration coefficients, $B_{m n}$.

Note that since $R_{0}^{0}(r)=1$, the Zernike polynomial basis contains the unit as an expansion term, which means that when illuminated by a plane wave of amplitude $E(r, \varphi)=$ const, the Zernike filter yields only one non-zero coefficient of the expansion in Eq. (10):

$$
\left|C_{00}\right|^{2} \neq 0
$$

From Eq. (6) it also follows that the diffraction orders corresponding to the basis functions with different numbers $\mathrm{m}$, but with the same numbers $n$, will have similar diffraction patterns (circular structures at $n>0$ ) in the Fourier-plane:

$$
\left|W_{n m}(\rho)\right|=r_{0}^{2} \frac{\left|J_{n+1}\left(k f^{-1} r_{0} \rho\right)\right|}{\left(k f^{-1} r_{0} \rho\right)} \text {. }
$$

The simulation parameters were as follows: 256 pixels on the radius $r$ and 256 pixels on the angle $\varphi$, $r_{0}=1 \mathrm{~mm}, k=10^{4} \mathrm{~mm}^{-1}, f=100 \mathrm{~mm}$. We designed a 25-channel filter [18] that generates diffraction orders for the basis functions with the numbers $(n, m): m \leq 8$ and $n \leq 8$, propagated at some angles to the optical axis.

Figure 2 depicts: (a) the half-tone Zernike filter phase (black colour corresponds to the phase value of 0 and white to $2 \pi$ ), (b) 25 diffraction orders generated in the lens frequency plane (negative), and (c) the correspondence between the numbers $(n, m)$ and diffraction orders. 


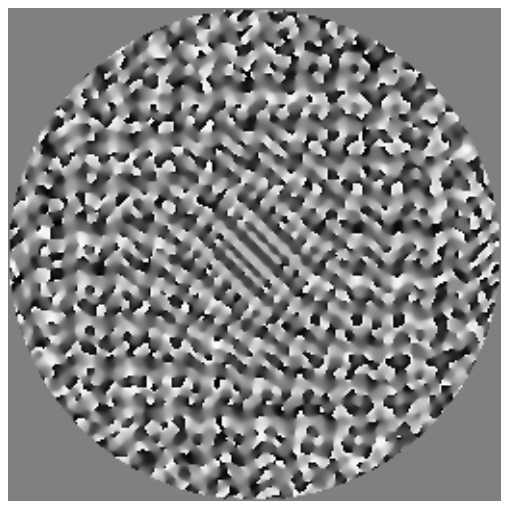

(a)

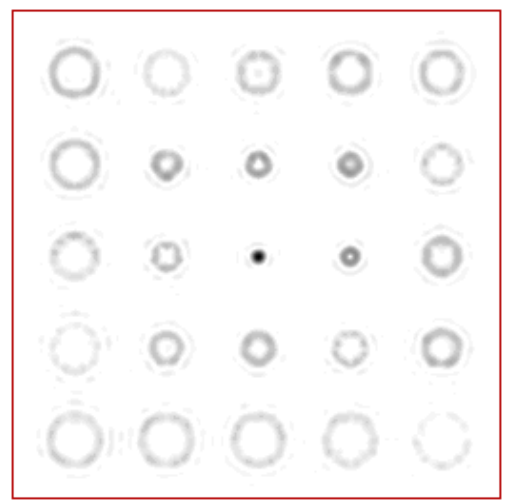

(b)

\begin{tabular}{|l|l|l|l|l|}
\hline$(7,1)$ & $(6,6)$ & $(6,4)$ & $(6,2)$ & $(6,0)$ \\
\hline$(7,3)$ & $(3,1)$ & $(2,2)$ & $(2,0)$ & $(5,5)$ \\
\hline$(7,5)$ & $(3,3)$ & $(0,0)$ & $(1,1)$ & $(5,3)$ \\
\hline$(7,7)$ & $(4,0)$ & $(4,2)$ & $(4,4)$ & $(5,1)$ \\
\hline$(8,0)$ & $(8,2)$ & $(8,4)$ & $(8,6)$ & $(8,8)$ \\
\hline
\end{tabular}

(c)

Fig. 2 (a) The half-tone phase of the Zernike filter, (b) the intensity distribution in the lens focal plane, and (c) the correspondence between the numbers $(n, m)$ and diffraction orders.

The filter is assumed to be illuminated by a plane wave. In this case, the analyser "splits" the incident beam onto 25 beams of approximately the same energy. $80 \%$ of the total illuminating beam energy is accounted for by these diffraction orders. From Fig. 2(b) the intensity is seen to be zero at all central points of the Fourier plane except for the zero order, $(0,0)$, so meaning that the illuminating wavefront is aberrationfree.

Figure 3 depicts the result of operation of the same 25-channel Zernike filter illuminated by the beam composed of three basis Zernike functions with the same weights and numbers, $(n, m):(2,0)+(5,3)+(7,7)$.
Shown in Fig. 3 are: (a) the illuminating beam intensity and (b) the diffraction pattern in the Fourier plane.

When compared with the distribution of mode numbers between the orders (Fig. 2(c)), Fig. 3(b) suggests that the intensity is non-zero (black spots in Fig. 3(b)) at the central points of the diffraction orders with the numbers $(2,0),(5,3)$, and $(7,7)$. Table 1 gives the corresponding averaged values in the vicinity of the central points for all orders (the vicinity size is $3 \times 3$ pixels).

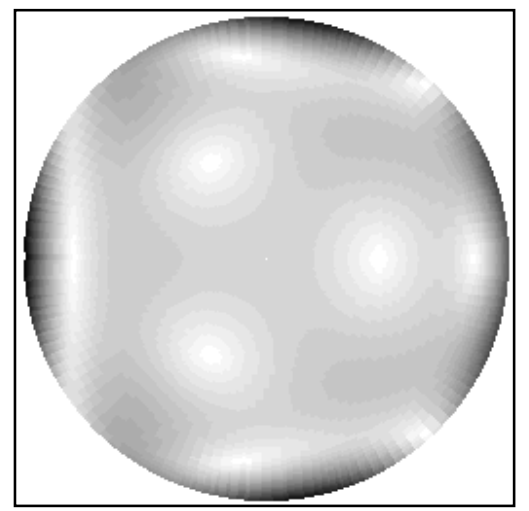

(a)

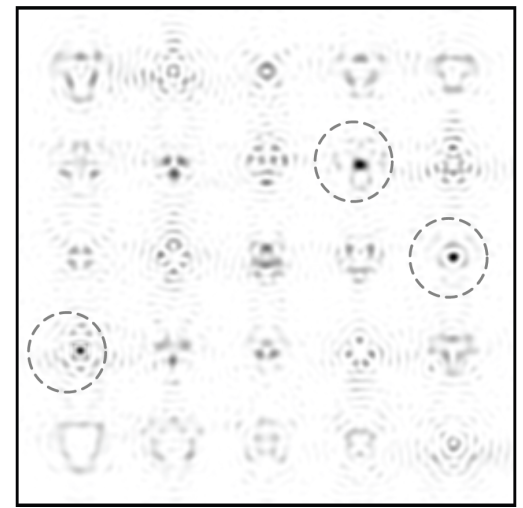

(b)

Fig. 3 Operation of the Zernike filter (see Fig. 2(a)): (a) the illuminating beam intensity and (b) the diffraction pattern in the Fourier plane.

Table 1 Averaged intensity values in the vicinity of central points for the Fourier-plane orders. The Zernike filter (Fig. 2(a)) is illuminated by a beam composed of three modes: $(2,0)+(5,3)+(7,7)$.

\begin{tabular}{|c|c|c|c|c|}
\hline $\begin{array}{l}(7,1) \\
0,011\end{array}$ & $\begin{array}{l}(6,6) \\
0,028\end{array}$ & \begin{tabular}{|l|}
$(6,4)$ \\
0,005
\end{tabular} & \begin{tabular}{|l|}
$(6,2)$ \\
0,006
\end{tabular} & $\begin{array}{l}(6,0) \\
0,035\end{array}$ \\
\hline $\begin{array}{l}(7,3) \\
0.103\end{array}$ & \begin{tabular}{|l}
$(3,1)^{0,013}$ \\
\end{tabular} & \begin{tabular}{|l|}
$(2,2)$ \\
0,018
\end{tabular} & $\begin{array}{l}(2,0) \\
0.977\end{array}$ & \begin{tabular}{|c|}
$(5,5)$ \\
0.008
\end{tabular} \\
\hline $\begin{array}{l}(7,5) \\
0,004\end{array}$ & \begin{tabular}{|l}
$(3,3)$ \\
0,007
\end{tabular} & \begin{tabular}{|l}
$(0,0)$ \\
0,059
\end{tabular} & \begin{tabular}{|l}
$(1,1)$ \\
0,003
\end{tabular} & $\begin{array}{r}(5,3) \\
1,000\end{array}$ \\
\hline $\begin{array}{l}(7,7) \\
0,683\end{array}$ & \begin{tabular}{|l|}
$(4,0)$ \\
0,056
\end{tabular} & $\begin{array}{l}(4,2) \\
0,000\end{array}$ & \begin{tabular}{|l|}
$(4,4)$ \\
0,001
\end{tabular} & $\begin{array}{l}(5,1) \\
0,001\end{array}$ \\
\hline $\begin{array}{l}(8,0) \\
0,004\end{array}$ & $\begin{array}{l}(8,2) \\
0,014\end{array}$ & $\begin{array}{l}(8,4) \\
0,018\end{array}$ & $\begin{array}{l}(8,6) \\
0,006\end{array}$ & $\begin{array}{l}(8,8) \\
0,026\end{array}$ \\
\hline
\end{tabular}


One can see from Table 1 that the coefficients with the same weights in the input beam of the Zernike analyser possess different weights at the output:

$$
\left|C_{20}\right|^{2}=0,977, \quad\left|C_{53}\right|^{2}=1,000, \quad\left|C_{77}\right|^{2}=0,683 .
$$

In addition, the intensity in the other orders is not zero. This is due to the fact that in an effort to obtain a purely phase filter in Eqs. (7) and (8), the amplitude is replaced by a constant value. Thus, the squared modules of coefficients at the Zernike analyser output are seen to have been measured with a relative error of $20 \%$. There are two ways for reducing the error: a more exact computation of the Zernike filter phase combining different algorithms [14] and more accurate localisation of the diffraction order centres in which the coefficient modules are measured. In this section, an averaging value on area of $3 \times 3$ pixels was used. Such approach is less demanding for localisation but leads to a greater error. In the following section, we use another algorithm for centres' localisation.

\section{Wavefront reconstruction using a multi- order Zernike filter}

If in image recognition it suffices to compute the modules of the coefficients expanded in some orthogonal basis, this procedure is insufficient when reconstructing the light field complex amplitude. The unique reconstruction of the light field also requires the knowledge of the phase of the expansion coefficients.

We can reconstruct the coefficient phases if to the filter in Eq. (8) some linear combination of neighbouring basis functions is added [19]:

$$
\begin{aligned}
& s_{n m}(r, \varphi)=\left\{\Psi_{n m}^{*}(r, \varphi)+\Psi_{n^{\prime} m^{\prime}}^{*}(r, \varphi)\right\} \times \\
& \times \exp \left[i k f^{-1} r \rho_{n m}^{\prime} \cos \left(\varphi-\theta_{n m}^{\prime}\right)+v_{n m}^{\prime}\right], \\
& p_{n m}(r, \varphi)=\left\{\Psi_{n m}^{*}(r, \varphi)+i \Psi_{n^{\prime} m^{\prime}}^{*}(r, \varphi)\right\} \times \\
& \times \exp \left[i k f^{-1} r \rho^{\prime \prime}{ }_{n m} \cos \left(\varphi-\theta^{\prime \prime}{ }_{n m}\right)+v^{\prime \prime}{ }_{n m}\right] .
\end{aligned}
$$

In this case, the light intensity in the additional channels corresponding to the Fourier-spectrum points with spatial frequencies $\left(\rho_{n m}^{\prime}, \theta_{n m}^{\prime}\right)$ and $\left(\rho^{\prime \prime}{ }_{n m}, \theta^{\prime \prime}{ }_{n m}\right)$ are as follows:

$$
\begin{aligned}
& S_{n m}=\left|C_{n m}\right|^{2}+\left|C_{n^{\prime} m^{\prime}}\right|^{2}+2\left|C_{n m}\right|\left|C_{n^{\prime} m^{\prime}}\right| \cos \left(\phi_{n^{\prime} m^{\prime}}-\phi_{n m}\right), \\
& P_{n m}=\left|C_{n m}\right|^{2}+\left|C_{n^{\prime} m^{\prime}}\right|^{2}+2\left|C_{n m}\right|\left|C_{n^{\prime} m^{\prime}}\right| \sin \left(\phi_{n^{\prime} m^{\prime}}-\phi_{n m}\right),
\end{aligned}
$$

thus allowing the derivation of the phases $\phi_{n m}$, for example, assuming $\phi_{00}=0$.

The recursive relationship for the sought-for phases may be written in the form

$$
\phi_{n^{\prime} m^{\prime}}-\phi_{n m}=\tan ^{-1}\left(\frac{P_{n m}-I_{n m}-I_{n^{\prime} m^{\prime}}}{S_{n m}-I_{n m}-I_{n^{\prime} m^{\prime}}}\right)
$$

Thus, the optical method under consideration makes it possible to find the complex coefficients of the light field expansion in terms of an orthogonal basis and to reconstruct this field.

We designed a 25-channel Zernike filter that can generate modes in different diffraction orders with the numbers $(n, m): n \leq 4$ and $m \leq 4$ (nine modes altogether) and their linear combination $(8+8$ altogether).

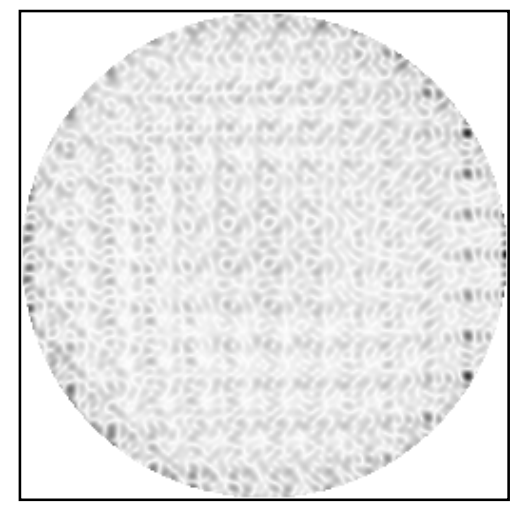

(a)

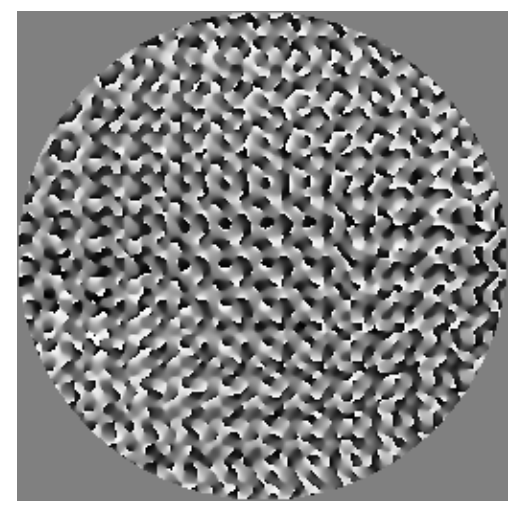

(b)

\begin{tabular}{|c|c|c|c|c|}
\hline S8 & S7 & S6 & S5 & S4 \\
\hline P1 & $(3,1)$ & $(2,2)$ & $(2,0)$ & S3 \\
\hline P2 & $(3,3)$ & $(0,0)$ & $(1,1)$ & S2 \\
\hline P3 & $(4,0)$ & $(4,2)$ & $(4,4)$ & S1 \\
\hline P4 & P5 & P6 & P7 & P8 \\
\hline
\end{tabular}

(c)

Fig. 4 (a) The half-tone amplitude and (b) phase of the Zernike filter, and (c) the distribution of modes numbered $(n, m)$ and their linear combinations between the orders. 
Figure 4 depicts (a) the half-tone amplitude and (b) the phase of the Zernike filter, and (c) the distribution of modes with the numbers $(n, m)$ and their linear combinations between the orders.

(a)

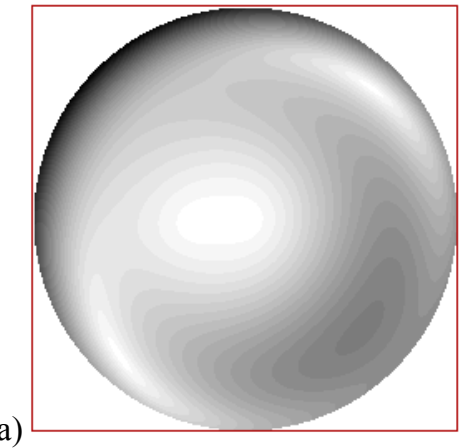

(b)

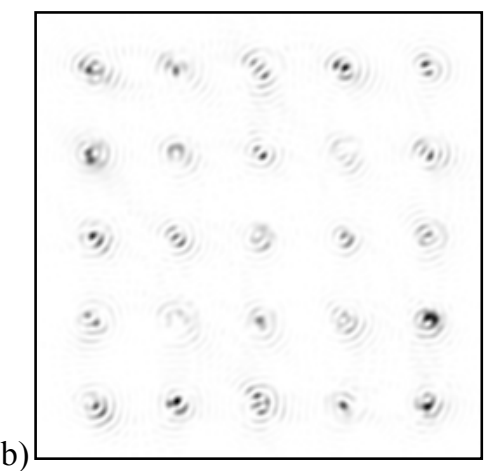

(c)

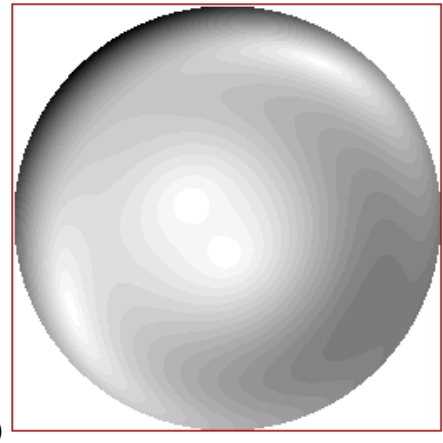

(d)

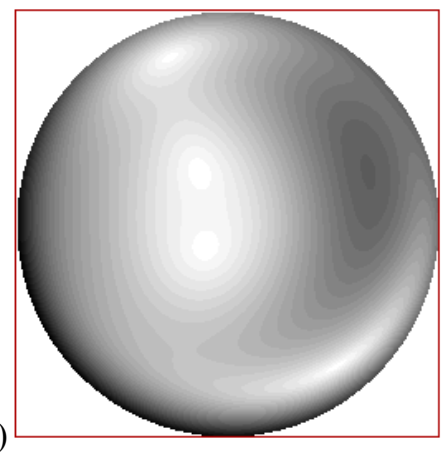

Fig. 5 (a) The intensity of the beam under analysis, (b) the diffraction pattern in the Fourier-plane produced by a purely-phase Zernike filter (shown in Fig. 4(a), (b)), (c) the light field reconstructed by an amplitude-phase filter, and (d) the light field reconstructed by the purelyphase filter.

Figure 5 depicts how such a 25-channel Zernike filter operates when illuminated by a beam composed of three modes with the coefficients $C_{n m}: C_{11}=\exp (i 0)$,
$C_{33}=\exp (i \pi / 2)$ and $C_{42}=\exp (i \pi)$. Shown in Fig. 5 are (a) the intensity of the beam under analysis, (b) the diffraction pattern in the Fourier plane for a phase-only filter, (c) the light field reconstructed by an amplitudephase filter, and (d) the light field reconstructed by the phase-only filter.

Intensities of expansion coefficients are shown in Table 2. To measure coefficients' values, we search the maximum value in the defined vicinity of diffractive orders' localisations. Such approach gives a smaller error of coefficients measurement than the averaging used in the previous section. In particular, for phaseonly filter the error has decreased from $20 \%$ to $9 \%$. Thus, it is shown that correct definition of positions of diffractive orders is very important for correct solution of the problem.

The example suggests that in a wavefront analysing, when it will suffice to measure the modules of expansion coefficients, a phase-only filter may be used to advantage. When diffractive orders are localised sufficiently correct the error in measuring the modules of the expansion coefficients is less than $9 \%$. At the same time, reconstructing the full information about the light field also requires the knowledge of the phase of the expansion coefficients. In this case, an amplitudephase filter should be used.

\section{Conclusions}

Multi-ordered diffractive element fitted with the set of Zernike polynomials was proposed to use for the direct measurement of the wavefront aberration coefficients of eye optical system.

Investigations suggest that in a wavefront analysing, when it will suffice to measure the modules of expansion coefficients, a phase-only filter may be used to advantage (the error in measuring the modules of the expansion coefficients is less than $9 \%$ when diffractive orders are localised sufficiently correct). At the same time, reconstructing the full information about the light field also requires the knowledge of the phase of the expansion coefficients. In this case, an amplitude-phase filter should be used.

Thus, it is shown numerically that multi-order phase diffractive elements allow to confidently and simultaneously detect several tens of expansion coefficients. We conducted simulation experiments with different coefficients corresponding to aberrations of both low and high orders. Therefore, we do not expect significant variations in the measurement error due to the presence of large number of modes in the wave front. This will enable to investigate insufficiently known high-order aberrations for the differentiated diagnostics of eye diseases.

\section{Acknowledgments}

The work was in part financially supported by the Russian Foundation for Basic Research (grant \#\#13-0700266, 14-29-07133, 15-29-03823) and the Ministry of education and science of the Russian Federation. 
S.N. Khonina et al.: Zernike phase spatial filter for measuring the aberrations of the optical structures of the eye

Table 2 Results of reconstructing the complex coefficients by an amplitude-phase and phase-only filters.

\begin{tabular}{lllllll}
\hline Coefficients & $\left|C_{11}\right|^{2}$ & $\left|C_{33}\right|^{2}$ & $\left|C_{42}\right|^{2}$ & Error & $\phi_{33}-\phi_{11}$ & $\phi_{42}-\phi_{33}$ \\
\hline \multicolumn{1}{|l}{ Initial } & 1 & 1 & 1 & & 1,57 & 1,57 \\
\hline $\begin{array}{l}\text { Reconstructed by } \\
\text { the amplitude- } \\
\text { phase } \\
\text { filter }\end{array}$ & 0,272 & 0,274 & 0,279 & $3,1 \%$ & 1,46 & 1,54 \\
\hline $\begin{array}{l}\text { Reconstructed by } \\
\text { the phase-only } \\
\text { filter }\end{array}$ & 12,12 & 14,83 & 14,59 & $8,6 \%$ & 0,95 & 1,53 \\
\hline
\end{tabular}

